\title{
ANALYTIC MODELING OF A SIZE-CHANGING SWIMMER
}

\author{
Gabriel D WEYMOUTH ${ }^{1}$, Francesco GIORGIO-SERCHI ${ }^{2}$ \\ ${ }^{1}$ University of Southampton, Faculty of Physical Sciences and Engineering \\ Boldrewood Campus, Southampton, UK \\ ${ }^{2}$ Second author affiliation
}

\begin{abstract}
.
Cephalopods use large-scale structural deformation to propel themselves underwater, changing their internal volume by $20-50 \%$. In this work, the hydroelastic response of a swimmer comprised of a fluid-filled elastic-membrane is studied via an analytic formulation of two coupled non-linear dynamic equations. This model of the self-propelled soft-body dynamics incorporates the interplay of the external and internal added-mass variations. We compare the model against recent experiments for a body which abruptly reduces its cross-section to eject a single jet of fluid mass. Using the model we study the impact of size-change excitation on sustained swimming speeds and efficiency.
\end{abstract}

Key words: fluid-structure interaction, biological flow, swimming, modeling.

\section{Introduction}

The maritime sector requires complex tasks to be performed in always more forbidding environments and with a constantly increasing degree of autonomy. These requirements are fostering the incremental improvement of the manoeuvring capabilities of state-of-the-art underwater vehicles [13, 2, 14] either by refining navigation and positioning systems (see Hover et al., 2012) or by undertaking completely disruptive design processes. This is the case of biomimetics, where water dwelling organisms are taken as the source of inspiration for the development of innovative vehicles. Underwater robotics has extensively employed the swimming biomechanics of fish and other aquatic creatures in order to endow new prototypes with the capability of hovering, short radius turning, fast start/slowdown and low-speed manoeuvring $[9,18,1]$. The propulsion routines of biologically- inspired robots entails, for the most part, cyclic oscillations of one or more body parts. These commonly drive the onset of momentum-rich vortical flow structures responsible for generating unsteady hydrodynamical forces which propel the vehicles. Flapping foil propulsion, for instance, relies on actuators which mimic the continuous deformation of fins and tails by means of discrete sequences of rigid links and joints. However, new actuators 


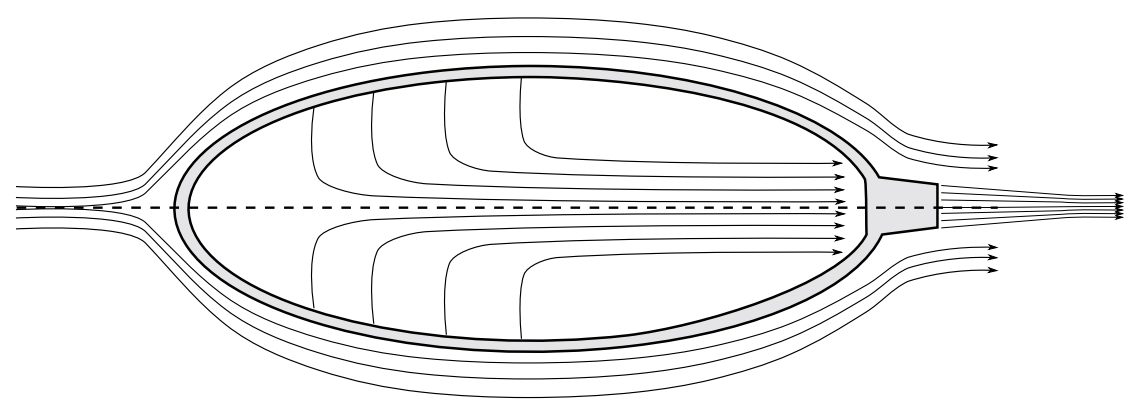

Figure 1: Flow kinematics inside and outside a self-propelled hollow membrane oscillating around its equilibrium configuration.

which more closely resemble the compliant nature of living tissues are now being developed by exploiting soft structures [10] which offer the advantage of intrinsic safer physical interaction with the environment as well as a higher degree of agility $[17,11]$.

The employment of compliant body parts or entirely soft-bodied vehicles and actuators not only provides a higher degree of structural resilience and the need for less-refined control strategies, but it also lends itself to mimic those organisms which rely extensively on large body-volume variations to propel themselves, such as squids and octopi. These sea-dwelling creatures sport a repertoire of extremely aggressive manoeuvres thanks to their pulsed-jetting propulsion which is driven by the inflation and deflation of a cavity of their body [7]. While the study on pulsed-jet locomotion has mainly revolved around the contribution to thrust from the vortex generated at the nozzle exit-plane [8], it is becoming apparent that the role of the external shape variation represents a prominent factor in this mode of propulsion, [15, 3]. The analysis performed on submerged bodies subject to abrupt shape-changes confirms that the forces associated with added-mass variation participate in the generation of thrust to a large extent, [16] [6]. Thus, the exploitation of added-mass variation as a potential source of thrust can have a major impact on the design (Giorgio-Serchi et al., 2016) [3] and control [4, 12] of new kinds of underwater vehicles. To this end, in this paper we devise a model for capturing the coupled fluid-structure-interaction effects of such kind of vehicles. The purpose of this model is to offer a fast tool for exploring the design space of new prototypes and shed light over the potential to employ added-mass variation effect as a source of thrust in aquatic vehicles.

\section{Methodology}

We consider a canonical self-propelled size-changing swimmer consisting of a hollow ellipsoidal neutrally buoyant elastic shell completely immersed in fluid, Figure 1. In the manner of squids and cephalapods, the swimmer propels itself by contracting its cross section, characterized by the minor semi-axis $B$, while maintaining the major semi-axis length $L$ fixed, and ejecting a portion of the internal fluid mass through an exit nozzle with area $A_{e}$. For sustained propulsion, the shell re-inflates with fluid via an opening at the center of mass to avoid ambiguity, and repeats the process. 


\subsection{Coupled swimming model}

The length and time scales of the body are set by the invariant major semi-axis length $L$ and frequency of pulsation $\omega$. The mass scale is set by the water density $\rho$. We describe the dynamics of this system with two-degrees of freedom; the body's dimensionless translation velocity $u=U /(L \omega)$ and dimensionless minor semi-axis length $b=B / L$.

Using a superscript notation to denote the coefficients related to each of the two modes, the dynamical equations are expressed as

$$
\begin{gathered}
m^{(u)} \dot{u}+c^{(u)} u=\tau \\
m^{(b)} \ddot{b}+c^{(b)} \dot{b}+k^{(b)}\left(b-b_{0}\right)=\psi
\end{gathered}
$$

where $m, c, k$ are the dimensionless lumped inertia, damping, and stiffness coefficients for the translation and deformation, $\tau$ is the jet thrust, $b_{0}$ is the resting semi minor-axis length and $\psi$ is the deformation excitation. The goal is to determine the form of these coefficients, making simplifications as appropriate.

\subsection{Translation model}

Building on previous work $([15],[5])$ the translation mode can be modeled as

$$
\left(M_{s}+M_{f}\right) \frac{d U}{d T}=\sum F=-\frac{d M_{f}}{d T} U_{j}-\frac{d}{d T}\left(M_{x x} U\right)-\frac{1}{2} C_{D} A_{f}|U| U
$$

where $M_{s}$ is the (constant) mass of the structure and $M_{f}$ is the (variable) mass of the internal fluid and $\sum F$ are the sum of the fluid contributions. The first fluid forcing term is the thrust due to mass flux out of the jet at speed $U_{j}$. The second term is the dynamic added-mass force based on the added mass in the translation direction $M_{x x}$. The third is a quasi-steady drag force based on the frontal area $A_{f}$ and an empirical drag coefficient $C_{D}$ for slender ellipsoids.

The kinematics of the system link the changes in vehicle volume $V=\frac{4}{3} \pi B^{2} L$ to the changes in internal mass and jet velocity.

$$
M_{s}+M_{f}=\rho L^{3}\left(\frac{4}{3} \pi b^{2}\right), \frac{d M_{f}}{d T}=\rho L^{3} \omega\left(\frac{8}{3} \pi b \dot{b}\right) \propto A_{e} U_{j}
$$

As stated above, we will consider the jet thrust to only be active during deflation. Similarly, the external added mass is modeled as that of the inscribed spherical body

$$
M_{x x}=\rho L^{3}\left(\frac{2}{3} \pi b^{3}\right), \frac{d M_{x x}}{d T}=\rho L^{3} \omega\left(2 \pi b^{2} \dot{b}\right)
$$

which is within $0.01 \rho L^{3}$ of the analytic added mass of an ellipsoid for all values of $B<2 L$. The variational term is critical because as the ellipsoid changes size during translation, the variation in added-mass produces a force $-\frac{d M_{x x}}{d T} U$, which significantly changes the dynamics of the swimmer. 
Grouping terms shows that the inertia of the translation mode is the sum of the hollow body mass, the mass of internal fluid, and the added-mass of the external flow. Translation damping includes forces from the fluid drag and the added-mass variation. Applying the dimensional scaling defined above gives the translation model coefficients as

$$
m^{(u)}=\frac{2}{3} \pi b^{2}(2+b), c^{(u)}=\pi b^{2}\left(2 \dot{b}+\frac{1}{2} C_{D}|u|\right), \tau=\alpha\left(\frac{8}{3} \pi b \dot{b}\right)^{2}
$$

where $\alpha=L^{2} / A_{e}$ during deflation and $\alpha=0$ during inflation. Note that each term is coupled to the deformation mode (via $b$ or $\dot{b}$ ), that the drag term is nonlinear (as expected), and that $A_{e}$ is the only free design parameter in the translation model.

\subsection{Deformation model}

As a first step toward modeling jetting swimmers, only the radial expansion mode of the membrane deformation is considered. Therefore the membrane remains ellipsoidal at all times ${ }^{1}$ and the radius $R$ along the body length is defined as

$$
R=B \sqrt{1-x^{2}}=L b \sqrt{1-x^{2}}
$$

where the body-fixed coordinate runs along $X=-L \ldots L$ and $x=X / L$. The governing equation for this mode is then

$$
\left(I_{s}+I_{f}\right) \frac{d^{2} B}{d T^{2}}+\left(C_{s}+C_{f}\right) \frac{d B}{d T}+\left(K_{s}+K_{f}\right) B=K_{s} B_{0}+\Psi
$$

where $I_{s}$ is the modal inertia of the structure and $I_{f}$ is the added inertia of the fluid due to acceleration of this mode. Similarly, $C_{s, f}$ and $K_{s, f}$ are the structural/fluid damping/restoring coefficients. $K_{s} B_{0}$ is the resting force needed to achieve a nonzero resting size $B_{0}$ and $\Psi$ is the excitation force. The structural coefficients depend on the design and material of the structure and will not be investigated in detail here. However, we will link the fluid coefficients to the kinematics of the swimmer, as we did for the translation model.

First, we investigate the added inertia by determining the fluid kinetic energy induced by deformation. Since the ellipsoid is streamlined, the confined internal flow causing the jet can be conceptualized as 1D, Figure 2. As is well known, confined flows amplify the kinetic energy for a given motion, and so the internal flow will be the dominant fluid contribution:

$$
E_{f} \approx \frac{1}{2} \rho \int U_{i}^{2} d V=\frac{1}{2} \rho L \int_{-1}^{x_{e}} \frac{F^{2}(x)}{A(x)} d x
$$

where $U_{i}$ is the internal flow velocity, $F$ is the flux through area $A=\pi R^{2}=$ $L^{2} \pi b^{2}(1-x)$ and $x_{e}<1$ is the exit location.

Incompressible flow requires that the change of the section area due to shrinking is equal to the increase in the flux through that section. Therefore the differential

\footnotetext{
${ }^{1}$ Note that if the length and exit size are fixed, we can't maintain a perfect ellipsoidal shape while shrinking. However, for modest size changes and small exit areas, the error will be small and confined to the region near the exit plane.
} 


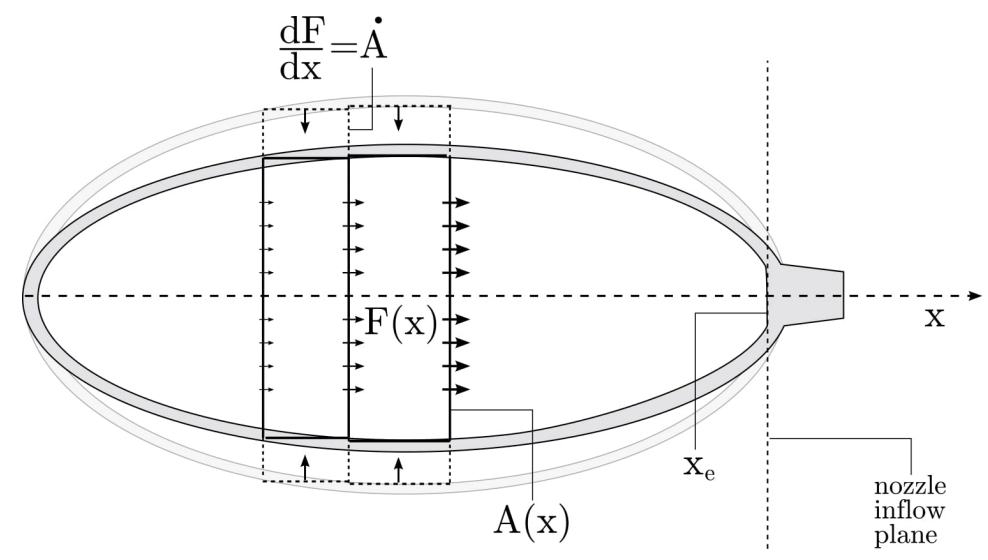

Figure 2: Relevant terms in the definition of the internal added-mass.

equation for $F$ is

$$
\frac{d F}{d X}=\frac{d A}{d T}=2 \pi L^{2} \omega_{n} b \dot{b}\left(1-x^{2}\right)
$$

And integration gives

$$
\frac{F(x)}{L^{3} \omega}=2 \pi b \dot{b} \int_{-1}^{x}\left(1-\tilde{x}^{2}\right) d \tilde{x}=\frac{2}{3} \pi b \dot{b}\left(2+3 x-x^{3}\right)
$$

Substituting $F$ and $A$ gives

$$
\begin{aligned}
\frac{1}{L^{4} \omega^{2}} \int_{-1}^{x_{e}} \frac{F^{2}}{A} d x & =\frac{4}{9} \pi \dot{b}^{2} \int_{-1}^{x_{e}} \frac{\left(2+3 x-x^{3}\right)^{2}}{1-x^{2}} d x \\
& =\left.\frac{4}{9} \pi \dot{b}^{2}\left(-4 x+2 x^{2}+\frac{5 x^{3}}{3}-\frac{x^{5}}{5}-8 \log (x-1)\right)\right|_{-1} ^{x_{e}}
\end{aligned}
$$

We can simply this formula by using $x_{e} \approx 1$ for the nonlinear polynomial parts. The fluid inertia, defined by $E_{f}=\frac{1}{2} I_{f}\left(\frac{d B}{d T}\right)^{2}$, is therefore

$$
\frac{I_{f}}{\rho L^{3}}=\frac{16}{9} \pi\left(2 \log \left(\frac{2}{1-x_{e}}\right)+x_{e}-\frac{34}{15}\right)
$$

As an example, if the body is slender with $b=0.2$ and $x_{e}=0.8$ then $I_{f} \approx 17 \rho L^{3} \approx$ $100\left(M_{s}+M_{f}\right)$ ! It is clear that this term greatly dominates over structural inertia for a density matched body.

Similarly, the fluid damping term will also be dominated by the internal flow. We model this loss using a Darcy friction factor. The pressure head loss at the jet exit and due to wall shear stresses approaching the jet can be written:

$$
\Delta p=\frac{1}{2} \rho C_{F} U_{j}\left|U_{j}\right|
$$

where $C_{F}$ is Darcy's friction factor

$$
C_{F}=\frac{32}{R e} \frac{1}{\sqrt{1-x_{e}^{2}}}+\frac{1}{2}
$$


which comprises of the term for head loss along constant radius cylindrical pipes at $\operatorname{Re}<2100$ and of an additional coefficient which accounts for viscous effect at square-edged inlets.

Finally, we consider if there are any fluid restoring forces. There is no fluid restoring force if $u=0$ since the fluid is static regardless of the value of $b$. However, when the body is in motion the pressure distribution on the external surface depends on the shape, and therefore $b$. This scaling argument shows that $k_{f}=K_{f} /\left(\rho L^{3} \omega_{n}^{2}\right) \propto-u^{2} b$, therefore its relative importance is quantified by a Weber number $W e=u^{2} b / k_{s}$. The dynamics of the fluid and body are strongly coupled at high $W e$ and the structural model will require more than a single deformation mode. We assume $W e \ll 1$ for the slender and stiff elastic body considered in this work.

Using these approximations, modal deformation coefficients are dominated by the internal flow and structural stiffness, ie

$$
m^{(b)}=I_{f} / \rho L^{3}, c^{(b)}=\frac{1}{2} C_{F}|\dot{b}|, k^{(b)}=k_{s}
$$

Note that in this case the deformation equation is decoupled from $u$ and when the deformation amplitude $|b|=\max (b)-b_{0}$ is small, we have an effectively linear oscillator with natural frequency $\omega_{n}=\sqrt{k_{s} / m^{(b)}}$ and damping ratio $\zeta=\frac{2}{3 \pi} C_{F} \omega|b| / \sqrt{m^{(b)} k_{s}}$.

\subsection{Jetting escape model validation}

Next our dynamical model is compared to the experimental results of Weymouth, Subramaniam, and Triantafyllou, Bioinspiration and Biomimetics, 2015. In that work, an elastic membrane was stretched over a rigid hull, inflated with water, and allowed to freely translate as it deflated. The body jetted away at high-speed, similar to the escape maneuver of an octopus. The experimental vehicle has $L=18 \mathrm{~cm}$, $A_{e} / L^{2}=0.05$ and $b_{0}=0.2$ and the initial mass was 3.5 times the resting mass $M_{f}=3.5 M_{0}$, and the stiffness $k_{s}$ is set to achieve approximately the same emptying time as the experiment $T \approx 1 \mathrm{~s}$. These model was set to match these values and $C_{D}=0.05$ and $C_{F}=0.25$ are chosen from the literature (and did not significantly impact the results). Finally, the excitation $\psi$ is set to zero and the model equation 1 is integrated numerically.

An important difference between the analytic model described above and the experiment is that the vehicle's rigid endo-skeleton causes the ejection period to abruptly stop at $1.0 \mathrm{~kg}$ limit. Instead, the current model allows the shell to oscillate around the equilibrium width $b_{0}$, the point where the fluid content in the shell is about 1.0 $\mathrm{kg}$.

Despite these differences the comparison in Figure 3 is remarkably good. The model results replicate the essential acceleration curve, as well as the measured peak velocity. This model enables us to estimate the role of thrust and added-mass variation effect in determining the burst of acceleration of the body. In agreement with the postulated mechanics in Weymouth et al. (2015), the contribution from added-mass variation approaches $25 \%$ of the jet force on the vehicle. 

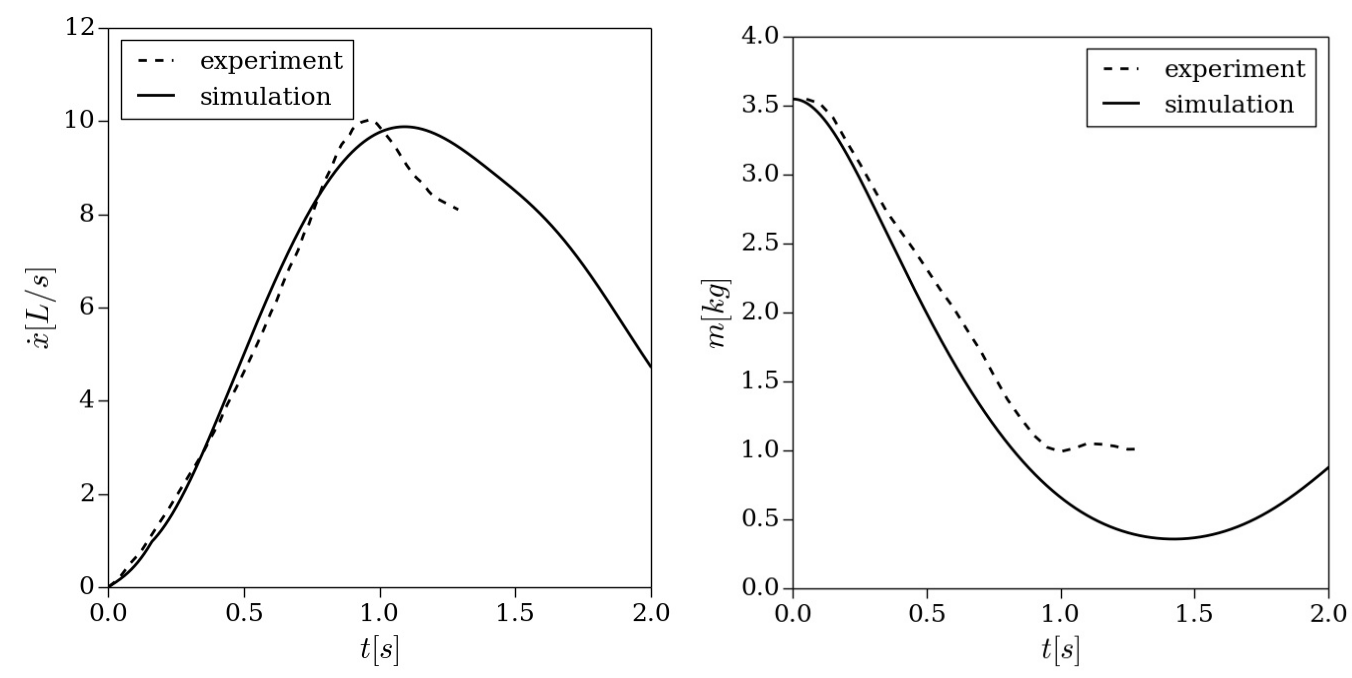

Figure 3: Comparison of the analytic model of equation 1 to to the experimental measurements for the translation speed $\dot{x}=U / L$ (left) and the internal mass $m \approx \rho \frac{4}{3} \pi L^{3} b^{2}$ (right).

\section{Pulsed Swimming Results}

These results give some confidence in the model to predict swimming performance. The next step is to use the model to study the impact of the excitation $\psi$ to the pulsed swimming characteristics.

In this set of tests we maintain the same parameters as in the validation test, but define the excitation as

$$
\psi=\|\psi\| \cos (\omega t)
$$

where the amplitude $\|\psi\|$ is chosen to achieve a characteristic deformation of $\delta b=$ $b-b_{0}=0.025$, ie $\|\psi\|=0.025 k_{s}$. This amplitude is somewhat arbitrary, but the corresponding volume change of $50 \%$ is similar to the levels found in nature.

Figure 4 shows the results as the excitation frequency is varied with respect to the swimmer's natural frequency in water. As with the validation case, we've initialized the swimmer at $350 \%$ of its resting volume and so we see the same initial peak in the velocity response. However, the long term behavior of the swimmer is determined by the frequency ratio $\omega / \omega_{n}$. When the ratio is greater than one, the amplitude of deformation and therefore the magnitude of velocity variation decreases. In contrast, when $\omega / \omega_{n}<1$ the deformation magnitude remains fairly large, but the jet velocity is reduced, leading to an overall decrease in average swimming velocity down to $\bar{u}=2.5 \mathrm{~L} / \mathrm{s}$. Excitation at the natural frequency generates the largest deformation as expected, and maintains a swimming speed of $\bar{u}=4 \mathrm{~L} / \mathrm{s}$.

A similar trend is observed in terms of the efficiency. As the body is freely swimming, the thrust and drag forces are balanced on average making the net force an inappropriate metric for the efficiency. Instead, the quasi-propulsive efficiency is 

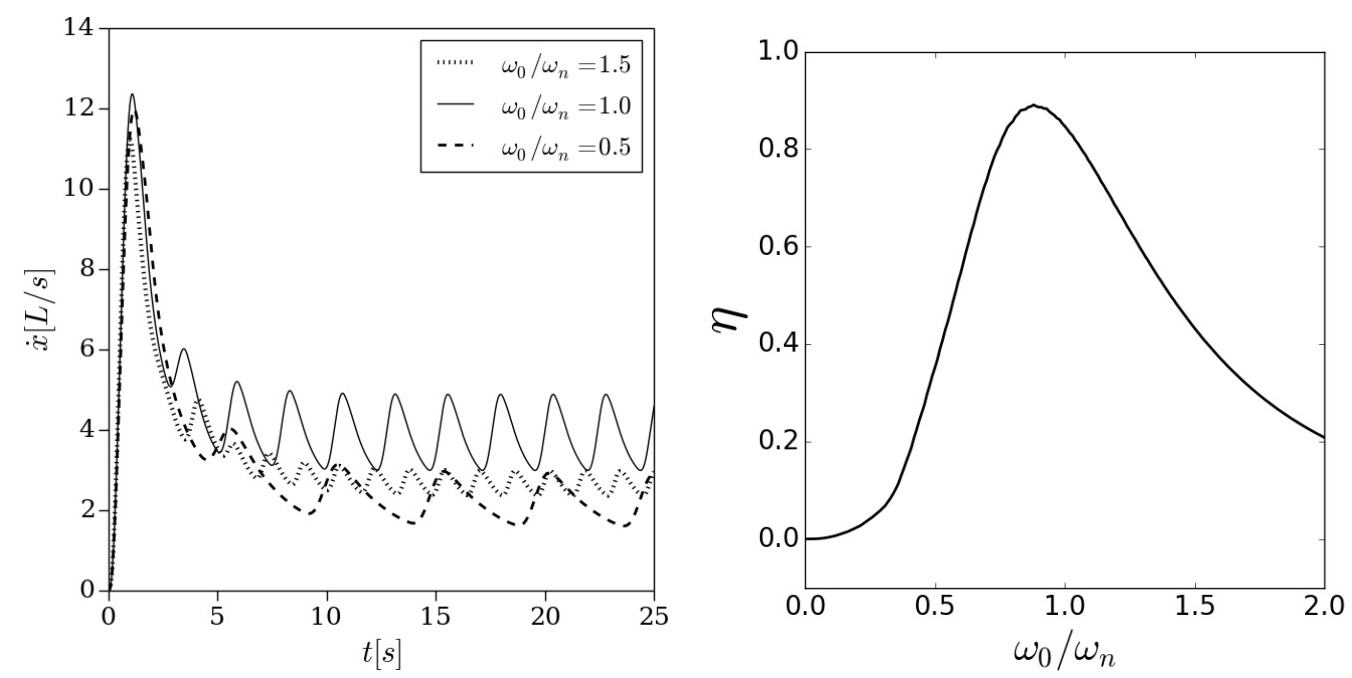

Figure 4: Performance of the size changing swimmer based on the coupled analytic model equation 1 . The velocity $\dot{x}$ over time (left) and the quasi-propulsive efficiency $\eta$ (right) as the excitation frequency ratio is varied.

used

$$
\eta=\frac{\bar{P}_{\text {in }}}{\bar{P}_{\text {tow }}}
$$

where $P_{\text {in }}=\psi \dot{b}$ is the power put into the motion by the excitation force, $P_{\text {tow }}=$ $\sum f u$ is the power it would take to tow the body at its resting size through the same velocity history. Both of these powers are averaged over a steady cycle. The result in Figure 4 shows that it is far more efficient to swim when exciting at the natural frequency, with the peak value reaching just above $90 \%$ for the chosen model parameters.

\section{Conclusions}

This study has developed a simplified analytic model for size changing swimmers. The model uses only two degrees of freedom, translation and radial deformation, but is found to capture the essentially features of previous experiments, namely:

1. The translation of an impulsive swimmer during an escape maneuver was found to reach peak velocities above $10 \mathrm{~L} / \mathrm{s}$.

2. The translation model accounts for the variation in added mass which produces a thrust approaching 25

In addition, the model highlights previously unexplored features related to the deformation, namely:

1. The deformation model accounts for the internal inertia of the fluid, which 
completely dominates over the structural inertia and so sets the natural frequency of the swimmer.

2. The deformation mode was found to be effectively uncoupled from the translation mode for stiff membranes $\left(k_{s}>>u^{2} b\right)$. Since the inertia of the flooded systems is high, large oscillation frequencies would only be possibly with stiff membranes, making this an important case to consider.

The model was then applied to the case of a swimmer which is excited in its deformation mode. The results show that the excitation at the natural frequency results in a mean swimming speed of $4 \mathrm{~L} / \mathrm{s}$ and a quasi-propulsive efficiency of $90 \%$. Excitation off of the natural frequency resulted in a rapid drop-off in efficiency and swimming speed.

Despite these promising results, there are still many issues to improve in this model. The fundamental weakness is in limiting the structure to a single radial deformation mode shape. In addition, visco-elastic damping in the structure may be important, depending on the manner of the swimmer's design. These elements and others are part of ongoing work, with the goal of developing a freely swimming experimental prototype in the near future.

\section{References}

[1] J. E. Colgate and K. M. Lynch. Mechanics and control of swimming: a review. IEEE Journal of Oceanic Engineering, 29:660-673, 2004.

[2] J. Elvander and G. Hawkes. Rovs and auvs in support of marine renewable technologies. In MTS/IEEE Oceans 2012, pages 1-6, Hampton Roads, VA, 2012.

[3] F. Giorgio-Serchi, A. Arienti, and C. Laschi. Underwater soft-bodied pulsed-jet thrusters: Actuator modeling and performance profiling. International Journal of Robotics Research, 2016.

[4] F. Giorgio-Serchi, F. Renda, M. Calisti, and C. Laschi. Thrust depletion at high pulsation frequencies in underactuated, soft-bodied, pulsed-jet vehicles. In MTS/IEEE OCEANS, Genova, Italy, May 2015.

[5] F. Giorgio-Serchi and G. D. Weymouth. Drag cancellation by added-mass pumping. Journal of Fluid Mechanics, 798, 2016.

[6] F. Giorgio-Serchi and G. D. Weymouth. Underwater soft robotics, the benefit of body-shape variations in aquatic propulsion. In Soft Robotics: Trends, Applications and Challanges, volume 17 of Biosystems \& Biorobotics, pages 37-46. Springer, 2016.

[7] W. Johnson, P. D. Soden, and E. R. Trueman. A study in met propulsion: an analysis of the motion of the squid, Loligo Vulgaris. Journal of Experimental Biology, 56:155-165, 1972.

[8] M. Krieg and K. Mohseni. Modelling circulation, impulse and kinetic energy of starting jets with non-zero radial velocity. Journal of Fluid Mechanics, 79:488-526, 2013.

[9] S. Licht, V. Polidoro, M. Flores, F. Hover, and M. Triantafyllou. Design and projected performance of a flapping foil auv. IEEE Journal of Oceanic Engineering, 29:786-794, 2004.

[10] Andrew D. Marchese, Cagdas D. Onal, and Daniela Rus. Autonomous soft robotic fish capable of escape maneuvers using fluidic elastomer actuators. Soft Robotics, 1:75-87, 2014. 
[11] Alexander Mörtl, Martin Lawitzky, Ayse Kucukyilmaz, Metin Sezgin, Cagatay Basdogan, and Sandra Hirche. The role of roles: Physical cooperation between humans and robots. The International Journal of Robotics Research, 31:1656-1674, 2012.

[12] F. Renda, F. Giorgio-Serchi, F. Boyer, and Laschi C. Modelling cephalopod-inspired pulsed-jet locomotion for underwater soft robots. Bioinspiration \& Biomimetics, 10, 2015.

[13] J. Vaganay, L. Gurfinkel, M. Elkins, D. Jankins, and K. Shurn. Hovering autonomous underwater vehicle-system design improvements and performance evaluation results. In International Symposium on Unmanned Untethered Submarine Technology, Durham, USA, august 2009.

[14] I. Vasilescu, C. Detweiler, M. Doniec, D. Gurdan, S. Sosnowski, J. Stumpf, and D. Rus. Amour v: A hovering energy efficient underwater robot capable of dynamic payloads. The International Journal of Robotics Research, 29:547-570, 2010.

[15] G. Weymouth and M.S. Triantafyllou. Ultra-fast escape of a deformable jet-propelled body. Journal of Fluid Mechanics, 721:367-385, 2013.

[16] G.D. Weymouth, V. Subramaniam, and M.S. Triantafyllou. Ultra-fast escape maneuver of an octopus-inspired robot. Bioinspiration \& Biomimetics, 10:1-7, 2015.

[17] Roger Woodman, Alan F.T. Winfield, Chris Harper, and Mike Fraser. Building safer robots: Safety driven control. The International Journal of Robotics Research, 31:1603-1626, 2012.

[18] J. Yu, R. Ding, Q. Yang, M. Tan, W. Wang, and J. Zhang. On a bio-inspired amphibious robot capable of multimodal motion. IEEE Transactions on Mechatronics, PP:1-10, 2011. 